\title{
Eigenartiges Exanthem in einem Falle von Miliartuberkulose bei chronischer myeloider Leukämie.
}

\author{
Von \\ Dr. Wilhelm Lutz.
}

(Aus der Dermatologischen Klinik Basel [Vorsteher: Prof. Lewandowsky].)

Krankheitsfälle von myeloider Leukämie, in deren Verlauf als Komplikation eine allgemeine Miliartuberkulose aufgetreten ist, sind schon mehrfach beobachtet worden; in keiner der mir zugänglichen Arbeiten finde ich aber eine Angabe, daß dabei auch die Haut Erscheinungen dargeboten habe.

Da jede der beiden Erkrankungen an sich schon zu Hauteruptionen führen kann, so kann unter Umständen ein Exanthem, das bei einer Kombination der beiden auftritt, ganz eigenartige Verhältnisse bieten, die für den Untersucher je nachdem gar nicht leicht zu deuten sind.

In dieser Hinsicht dürfte der nachstehende Fall, den wir an der hiesigen medizinischen Klinik beobachten konnten, von Interesse sein.

Seine.Krankengeschichte, für deren Überlassung ich Herrn Professor Stähelin bestens danke, lautet folgendermaßen:

Frau Paula B.-R., 37 Jahre. Eintritt in die med. Klinik am 30. XII. 1916. (K. G. 50/1917.)

An am nese: Vater und Mutter leben gesund, beide 62 jährig, 3 Geschwister gesund, eins †, einjährig, an Gehirnentzündung. Pat. hatte als Sängling Augenentzündung, als Kind Rotsucht und Keuchhusten. Sonst gesund. Sie ist seit 10 Jahren verheiratet, Mann und 5 Kinder gesund. Eine Frühgeburt nach dem 3. Kinde. Pat. hat seit Mai 1916 das Gefühl, daß etwas an ihr zehre, ohne bestimmte Beschwerden. Im Juli 1916 schien der Leib, besonders unter dem Rippenbogen, etwas geschwollen. Diese Schwellung nahm allmählich zu. Keine Schmerzen. Abnahme der Körperkraft. Mitte Dezember mußte sich Pat. zu Bett legen. Sie trat am 27. XII. ins Frauenspital ein. Stechende Schmerzen auf der linken Schulter beim Bewegen des Armes, nicht beim Atmen. Am 30. XII. auf innere Klinik verlegt. Appetit gut, Schlaf gering, Stuhlgang regelmäßig, Diurese o. B., Menses begannen mit 13 Jahren, regelmäßig, nicht stark, seit Herbst 1916 Metrorhagien, abundant und stets zu früh eintretend. Letzte Periode 21-27. XII. 1916. Seit 14 Tagen schwitzt Pat. stark, besonders nachts. Stets feuchte Haut.

Status praesens: Mittelgroße Frau, Haut blaß, etwas gelblichgrau, feucht, warm. Auf den Wangen circumscripte Rötung. Augen: Strabismus divergens concomitans sin. Links centr. Maculae corneae. Pupillen o. B. Hals schlank, Thyreoidea nicht vergrößert. Keine Lymphdrüsen vergrößert. Keine Supraclaviculardrüsen. Starke Pulsation der Carotiden und im Jugulum. Thorax sym- 
metrisch, Atmung oberflächlich und beschleunigt. Longengrenzen hinten rechts 3 Finger höher als links, sonst o. B. Wirbelsäule vom 1.-5. Dorsalwirbel Sförmig skoliotisch. Herz normal, erster Ton an der Spitze unrein, Puls regelmäßig, beschleunigt, leicht unterdrückbar.

Musc. trapez. stark druckempfindlich. Abdomen stark über Thoraxniveau erhaben, größter Umfang $94 \mathrm{~cm}$. Zahlreiche alte Striae der Bauchhaut. Der Leib links seitlich stark vorgetrieben, seitlich links vom Nabel gegen die Inguines hin ein Tumor palpabel mit hartem Rand, nicht druckempfindlich. Abhängige Partien nicht gedämpft. Leberdämpfung 3 Querfinger unter Rippenbogen. Rand palpabel, nicht druckempfindlich. Inguinal, Cubital, Axillardrüsen nicht vergrößert. Extremitäten und Reflexe o. B. Im Urin Diazo stark positiv. Temperatur erste 10 Tage um $38^{\circ}$, nachher zwischen $35^{\circ}$ and $37^{\circ}$ mit etwas remittierendem Typus.

Verla uf: 3. I. Blutstatus: Hämoglobin 60/90. Weiße Blutkörperchen 40300 , rote Blutkörperchen 3,08 Millionen.

8. I. Weiße Blatkörperchen 38300 , davon Neutrophile 63,5\%, Myelocyten $28,5 \%$, Lymphocyten 4,5\%, Mastzellen 1,5\%, Eosinophile 1,5\%, Myeloblasten $0,5 \%, 2$ Normoblasten auf 300 weiße Zellen.

2. II. Beginn der Röntgentherapie.

6. II. Weiße Blutkörperchen 27 900, davon Neutrophile 52\%, Lymphocyten groß $6 \%$, kleine $17 \%$, große Mononucleäre 6\%, Übergangszellen 14\%, Eosinophile $1 \%$, Mastzellen 4\%, Anysocitose.

14. II. Milztumor bedeutend zurückgegangen. Rand 1 Querfinger oberhalb des Nabels.

20. II. Pat. steht auf, fühlt sich wohl. Gewichtszunahme. Weiße Blut körperchen 45200 .

2. III. Leukocyten 39600 . Klagt über Halsweh.

14. III. Leukocyten 9100 bei mehrmaliger Zäblung.

17. III. 5600 Leukocyten, davon Neutrophile $83 \%$, Lymphocyten $22 \%$, Mononucleäre 0,3\%, Übergangszellen 1,0\%, Eosinophile 0,06\%, Mastzellen 1,0\%, Myelocyten 1,0\%, Myeloblasten 0,6\%.

20. III. Allgemeinbefinden gut. Nicht mehr müde. Abdomen weich, Milz 3 Querfinger unter Rippenbogen. Entlassen.

Diagnose: Myeloische Leukämie; trotz hochgradiger Leber und Milzschwellung nur wenig Teukocyten.

Wiedereintritt: 7. I. 1918.

$Z$ wische nana mnese: Nach Spitalaustritt wohl. Besorgte die Haushaltung. Im Mai 1917 soll sich im Kopf unter starken Schmerzen ein Geschwür gebildet haben, das sich durch den Mund entleerte. Hohes Fieber, widerlicher Geruch. Im November 1917 dickerwerden des Halses. Vergrößerung der Halsdrüsen, erst rechts, dann links. Etwas Engigkeit beim Atmen, Sohluckbeschwerden. Gehörsabnahme. Seit August 1917 zeitweise stark juckender Ausschlag an Armen und Beinen. Seit Anfang Dezember bettlägerig, von Zeit zu Zeit Ohnmachtsanfälle, Atemnot. Zunehmende Schwäche, Appetit gering, Durst.

Stat. praes.: Haut blaß, zeigt besonders an der Streckseite der Extremitäten kleine hellrote Knötchen. Augen wie früher, Hals und Rachen o. B. Lymphdrüsen am Kieferwinkel mandelgroß, leicht druckempfindlich, am Halse beiderseits bis über walnußgroß, gesondert tastbar, wenig verschieblich, mit Unterlage und Haut aber nicht verwachsen, kettenförmig nach abwärts ziehend. Axillar- und Inguinaldrüsen nicht vergrößert. Thorax, Lungen, Herz o. B., Abdomen weich, Leberrand 2 Querfinger unter Rippenbogen, Milz ca. 4 Finger unter dem Rippenbogen, deutlich palpabel, Extremitätenreflexe o. B. 
Verlauf: 9. I. Weiße Blutkörperchen 1100-1200, 12. I. weiße Blutkörperchen $1700-1800$.

14. I. Deliriert, Temperatur andauernd um $38^{\circ}$. Lymphdrüsen am Hals scheinen sich rasch zu vergrößern. Exanthem stärker hyperämisch. Lunge, Dämpfong r. hinten unten A. G. vesic. unbestimmt, beiderseits hinten unten vereinzelte, mittelblasige, nicht klingende Rasselgeräusche.

15. I. Leukocyten 2150.

16. I. Somnolent. Lunge R. H. U. Schallverkürzung bis zum Angulus scapulae. A. G. bronchial, feines Reiben und wenig mittelblasige, nicht klingende R. G. Rachen auffallend trocken, stark gerötet. Große Schluckbeschwerden Pat. verweigert deswegen jede Nahrungsaufnahme.

Blutplattenkulturen und Blut in Bouillonkölbchen, steril bis am 22. I.

Ohrenuntersuchung: normale Verhältnisse.

Augenuntersuchung: nichts von Belang.

18. I. Starker Hustenreiz. Schleimig eitriger Auswurf, ohne Tuberkelbacillen.

20. I. Agglutination auf Typhus and Paratyphus negativ. Lungendämpfung R. H. U. viel ausgesprochener. Lautes Bronchialatmen, auch L. H. U. Bronchialatmen. Pleuraprobepunktion ergibt keine Flüssigkeit. Im Anschluß an die Punktion etwas Blut expektoriert. Im Sputum keine Tuberkelbacillen.

22. I. Leukocyten 2200, Polynucl. Neutrophile 22,5\%, Lymphocyten 16,5\%, Mononucleäre $5,0 \%$, Übergangszellen $6,5 \%$, Eosinophile 2,0\%, Neutrophile Myelocyten 47,5\%. Exitus.

23. I. Starke Verschlimmerung des Befindens. Starke Cyanose und Dyspnoe.

Soweit die Aufzeichnungen von interner Seite. Am 14. I. waren wir zur Beurteilung der Hauterscheinungen zugezogen worden und hatten folgenden Befund erheben können:

Ha utstatus: Die Haut des ganzen Körpers hat einen leicht matt gelblichen Ton und zeigt ein Exanthem, in folgender Verteilung und Form: Behaarter Kopf und Gesicht sind frei von Efflorescenzen. Auf den Wangen finden sich einzelne kleine Telangiectasien. Am Hals ist die Haut in der unteren Hälfte rechts, gegen die Schultern hin, braunrötlich verfärbt und leicht schuppend. AuBerdem findet sich auf beiden Seiten eine mittlere Zahl von kleinen, stecknadelkopfgroßen Knötchen, die z. T. gleichmäßig hellrot gefärbt sind, z. T. ein etwas blasseres Zentrum und einen intensiv roten, schmalen Streifen an der Peripherie aufweisen. Die Brust ist frei, am Abdomen sieht man nur 3-4 hellrote kleine Knötchen. Zahlreiche kleine Knötchen, teils gleichmäßig hellrot, teils mit intensiv rotem. Saum, sind ïber dem Rücken diffus ausgesät, zwischen ihnen liegen ebenso zahlreich kleinste, bei Glasdruck nicht verschwindende, hellrote Hämorrhagien. Besonders stark befallen sind die Arme und zwar vorwiegend auf der Streckseite. Diese sind dicht übersät mit den gleichen kleinen etwa stecknadelkopfgroßen Primärefflorescenzen, doch sind diese hier zu unregelmäßigen, 1-2 cm Durchmesser haltenden Plaques zusammengetreten, welche als feinhöckerige, z. T. leicht, z. T. nicht schuppende, hellrote, derbe Herde über die Hautoberfläche prominieren. Besonders stark ist das Zusammentreten an den Ellenbogen ausgesprochen, an denen ein fast handtellergroßer Herd sich findet. Weniger Efflorescenzen finden sich auf der Beugeseite, etwas gehäuft in den Ellbeugen. In der rechten Ellbeuge weisen manche zentral eine kleine, hellrote, auf Druck bleibende Hämorrhagie auf. Zwischen den papulösen Efflorescenzen finden sich am rechten Vorderarm auch kleine, nicht prominente Hämorrhagien, die z. T. ebenfalls in kleinen Gruppen zusammengestellt sind. Am linken Vorderarm sieht man keine Hämorrhagien, dagegen zwischen den hellroten frischen Efflorescenzen eine ganze 
Anzahl abgeblaßter, nur noch als gelbliche, beim Kratzen leicht schuppende Flecke erscheinender, rückgebildeter Knötchen und Plaques. Die Finger sind frei, mit Ausnahme einer diffusen, von einzelnen hämorrhagischen Stippchen durchsetzten Rötung am Rücken der Endphalanx des rechten Mittelfingers. Auf dem rechten Thenar nur noch an tiefliegenden Pigmentstippchen erkennbare abgeheilte Plaque, sonst Handflächen frei. Eine stärkere Häufung von Efflorescenzen findet sich wieder am Gesäß. Es handelt sich hier mehr um hellrote Flecken als um Knötchen. Zwischen ihnen sieht man wieder ebenso zahlreiche abgeheilte Flecken als braune, beim Kratzen leicht schuppende Pigmentfleckchen. Die Oberschenkel wieder ziemlich frei von Efflorescenzen. Über «den Knien stärkere Anhäufung hellroter, flacher Knötchen. Über den Unterschenkeln und Fußrücken eine mittlere Zahl kleinster, fast punktförmiger, zum größten Teil in Rückbildung begriffener Hämorrhagien.

Wir fanden somit ein universelles, etwas stärker an den Streckseiten der Extremitäten und am Rücken ausgeprägtes Exanthem von ziemlich einheitlicher Erscheinung. Neben einfachen Hämorrhagien bestanden etwa stecknadelkopfgroße, derbe Knötchen, z. T. gleichmäßig hellrot, z. T. mit leicht gelblichem Zentrum, z. T. mit zentraler Hämorrhagie, meist vereinzelt stehend, an den Armen mehr gruppiert. Daneben fanden sich im Rückgang begriffene, leicht schuppende z. T. nur als Pigmentfleckchen noch erkennbare Efflorescenzen, nirgends Narben.

Zur Ergänzung dieses Befundes konnten wir am 16. I. zwei Hautstückchen excidieren und histologisch untersuchen. Das eine enthielt eine größere und eine kleinere isolierte Papel, das andere entsprach einer mehr diffus infiltrierten kleinpapulösen Stelle. Nach Fixierung in Sublimat, Härtung in Alkohol und Einbettung in Paraffin bot der mit Hämalaun-Eosin gefärbte Schnitt folgendes Bild: Im Bereiche des Knötchens findet sich ein ziemlich gut abgegrenztes, im Papillarkörper und in den obersten Schichten des Stratum reticulare gelegenes zelliges Infiltrat, das die Epidermis unter Verstreichung der Papillen halbkugelig vorwölbt. Die Epidermis ist auf 3-4 Zellreihen verdünnt, die Intercellularlücken sind hier und da etwas erweitert und enthalten vereinzelte Leukocyten. Das Stratum granulosum ist nicht zu erkennen, die Hornschicht ist etwas verdickt und splittert ab. Das Infiltrat selbst durchsetzt diffus das Bindegewebe, das leicht ödematös ist. Gerade unter dem Epithel ist das Ödem stärker, so daß hier sich kleine Hohlräume gebildet haben, welche die Epidermis bläschenartig etwas vom Corium abheben. Das Infiltrat setzt sich zusammen aus Bindegewebezellen, z. T. klein, mit spindelförmigem intensiv gefärbtem Kern, z. T. größer, sternförmig, mit hellerem, rundlichem Kern; aus Lymphocyten, Plasmazellen und polynucleären Leukocyten in ungefähr gleicher Menge. Außerdem sieht man hier und da mittelgroße Zellen mit kräftig gefärbtem Kern und deutlichem Protoplasmasaum, die den hier und da in Gefäßen liegenden Myelocyten entsprechen. In diesem Infiltrat liegen ferner unregelmäßig zerstreut einzelne auffallend große z. T. läng- 
liche, unregelmäßige Zellen, mit homogenem, stark tingiertem, rotviolettem Protoplasma und exzentrisch gelegenem, bläschenförmigem Kern. Dieser entspricht in der Größe etwa einem Fibroblastenkern und enthält ein deutliches Chromatingerüst und Nucleolen; hier und da zeigte er eine Einschnürung, so daB er wie gelappt aussieht und hier und da erhält man fast den Eindruck von zwei nebeneinanderliegenden Kernen, mehrere aber finden sich nirgends sicher nachweisbar in einer Zelle. Zwischen den Infiltratzellen sieht man einzelne erweiterte Gefäße, z. T. leer, z. T. mit roten Blutkörperchen und nur mit wenigen kernhaltigen Elementen. In ihrer Umgebung sind Lymphocyten, Plasmazellen und einige myelocytenähnliche Zellen etwas dichter gehäuft. Im Zentrum des Knötchens sieht man kleine Häufchen freier roter Blutkörperchen und im gleichen Gebiet einzelne Zellen mit Karyorhexis. Die übrige Cutis zeigt außer einer geringgradigen Lymphocytenansammlung an einzelnen Gefäßen keine Veränderungen.

Bei Färbung nach Unna-Pappenheim färbt sich das Protoplasma. der großen Zellen hellrosa; es erscheint hier und da etwas vacuolär. Der Kern zeigt ein grünes Chromatinnetz und 2-3 rote Nucleolen.

Bei van Giesonfärbung treten besonders diese großen Zellen durch ihr graugrünlich gefärbtes Protoplasma deutlich hervor.

Das zweite Hautstückchen zeigt folgende Verhältnisse: Der Papillarkörper ist im ganzen Bereich der Plaque etwas ödematös verdickt, die Bindegewebezellen sind etwas gequollen und zwischen ihnen liegen vermehrt Lymphocyten diffus eingestreut. An einzelnen papillargefäßschlingen sind sie so ausgeprägt, daß sie kleine Zellherde bilden. Um alle sichtbaren Gefäße, die etwas erweitert sind, finden sie sich, mit polynucleären Leukocyten, Plasmazellen und einzelnen Myelocyten vermischt in etwas stärkeren Ansammlungen, die sogar hier und da kleinere Herdchen bilden, besonders an einigen Papillengefäßschlingen. An einigen Stellen sieht man dann weiter noch starke, ausgesprochene, zellreiche Infiltrate, die etwas mehr flächenhaft angelegt sind und sich wegen des erhöhten Gehalts der Cutis an Lymphocyten überhaupt etwas weniger scharf absetzten, als das erst beschriebene Knötchen. Auch das Ödem ist geringer als bei letzterem, so daß die Anhäufungen kompakter erscheinen. Sie bestehen vorwiegend aus Fibroblasten und Lymphocyten, mit einzelnen plasma- und myelocytenähnlichen und nur wenigen der beschriebenen großen Zellen. Sie sind von reichlich etwas erweiterten Blutgefäßen durchzogen, ohne Hämorrhagien. Die Epidermis ist auch hier zum Teil verdünnt, mit verstrichenen Papillen und etwas vorgewölbt. Die Hornschicht ist überall etwas verbreitert. Nur eines der Infiltrate bietet einen etwas anderen Anblick. Es handelt sich offenbar um ein in Rückbildung begriffenes Knötchen. Es läßt sich bei ihm ein peripherer Ring von gleichem Aufbau wie beim erst- 
beschriebenen Knötchen von einem Zentrum unterscheiden, das etwas zellärmer ist, jedoch zahlreiche der großen Zellen mit gut gefärbtem homogenem Protoplasma und bläschenförmigem, exzentrischem Kern mit gut gefärbtem Kerngerüst aufweist. Das Grundgewebe ist hier fast gefäßlos, seine Fasern sind etwas hyalin verändert und bilden ein feines Maschenwerk. Die Epidermis ist über dieser Stelle besonders verdünnt. Im Zentrum des Knötchens ist sie leicht dellenförmig eingesunken und von einer Schuppenkruste aus Epithelzellen und Leukocyten bedeckt. Unna-Pappenheim- und van Gieson-Färbung ergeben keine Besonderheiten. Die elastischen Fasern sind im Bereiche der Infiltrate $z$. T. etwas fragmentiert, hier und da auch ziemlich vermindert.

Den Papeln entspricht also ein ziemlich scharf umschriebener entzündlicher Prozeß mit Zellinfiltrat, Gewebsödem, Gefäßinjektion und hier und da einer kleinen zentralen Hämorrhagie. Das Infiltrat besteht vorwiegend aus Lymphocyten und Fibroblasten, mit wenigen polynucleären Leukocyten, als Myelocyten zu deutenden Zellen, und spärlichen Plasmazellen gemischt. Zwischen diesen fallen sehr große rundliche oder ovale Zellen auf mit bläschenförmigen, z. T. etwas gelapptem Kern und blaßgefärbtem Protoplasma. Meist liegen sie vereinzelt eingestreut, nur an der einen oder anderen Stelle sind mehrere etwas zusammengestellt. Eines der untersuchten Knötchen zeigt zentrale Abheilung und einen peripheren, noch entzündlichen Saum. Neben diesen Papeln besteht stellenweise eine diffuse Ausstreuung von Lymphocyten im Corium und eine leichte lymphocytäre, mit einzelnen Myelocyten gemischte Infiltration um alle Gefäße, die stellenweise, besonders an den oberen Enden einzelner Papillargefäßschlingen zu einer etwas zellreicheren, aus Lymphocyten, Myelocyten, Fibroblasten und einzelnen Leukocyten, also von rein entzündlichem Charakter, ausgebildet ist.

Leider genügten diese Befunde nicht zu einer Klärung des Krankheitsbildes und zu einer Diagnosestellung. Die Knötchen trugen, gegenüber den banal entzündlichen Zellanhäufungen wohl einen etwas ausgeprägteren Charakter, doch war er nicht derart, daß er einen Vergleich mit einem bekannten Prozeß ermöglicht hätte.

Im Vordergrund stand natürlich die Frage nach dem Zusammenhang. der Hauteruption mit der Leukämie.

Die spezifischen tumorförmigen Hautinfiltrate bei myeloider Leukämie, wie sie von Hindenburg, Hirsehfeld und Tobias, Schleip und Hildebrand, Bruusgaard, beschrieben worden sind, waren natürlich leicht auszuschließen. Die Myelocyten, die sich in dem Zellgemisch der Infiltrate vorfanden, waren zu wenig hervortretend und ließen sich nicht höher bewerten, als in dem Sinn, daß sie eben ent- 
sprechend ihrem Vorhandensein als Blutzellen auch an den Infiltraten teilhaben mußten.

Schwieriger war schon die Entscheidung, ob das Exanthem auf eine toxische von der Leukämie abhängige Ursache zurückzuführen sei. Hautveränderungen dieser Art sind bei myeloischer Leukämie gelegentlich, wenn auch nicht gerade häufig, beschrieben worden. So erwähnt Palta uf das Vorkommen von Blutungen, Abscessen und Furunkeln, Arndt Blutungen, Urticaria simplex, papulosa und papulovesiculosa, Furunkeln und Karbunkel. Ob letzterer auch prurigoartige Exantheme gesehen hat, geht aus der Wortfolge nicht ganz sicher hervor. Für einen solchen Zusammenhang wäre in unserem Falle das zu Beginn der Eruption zeitweise bestehende Jucken zu erwähnen. Burckhardt sah neben Abscessen flache krustöse Ulcerationen.

Diesen Beobachtungen entsprach unser Exanthem nicht völlig; kleinste Blutungen fanden sich allerdings in reichem Maße, die Papeln zeigten aber alle für urticarielle oder krustöse Gebilde viel zu wenig exsudativen Charakter. Absceßbildung oder Zerfall bestand nirgends.

Einzig in einem Fall von Schultze ist eine Beobachtung verzeichnet, die vielleicht mit unserer verglichen werden kann. Die allerdings sehr kurz dauernde klinische Beobachtung nennt zwar nur hämorrhagische Bläschen und Blasen, im Sektionsprotokoll findet sich aber dazu ,am Abdomen ein feines, papulöses, gelbrötliches Exanthem mit dazwischen liegenden miliaren Hämorrhagien" erwähnt. Eine spezielle histologische Untersuchung dieser Knötchen ist nicht angeführt. Es heißt nur gelegentlich, die kleinen rotgefärbten Knötchen der Haut hätten aus Hämorrhagien und einer aus Anhäufung von nur mononucleären Zellen (Myeloblasten oder Myelocyten) bestanden, wie auch bei den Blasen das Infiltrat des Grundes wesentlich aus diesen gebildet worden sei. Durch den histologischen Befund würde also auch dieser Fall von unserem abweichen.

Die Histologie des unsrigen ließ sich zum Teil mit der Annahme eines toxischen Agens ganz wohl vereinbaren. Die zahlreichen perivasculären, in manchen Papillen auch etwas stärker flächenhaft ausgedehnten Infiltrate von rein entzündlichem Charakter, mit der allerdings ungewöhnlichen, aber nicht überreichlichen, durch die Leukämie als Grundkrankheit genügend erklärten Beimischung von Myelocyten konnten durch jedes beliebige entzündlich wirkende Toxin erzeugt sein. Weniger dagegen befriedigte uns diese Annahme für die Erklärung der eigentlichen Papeln. Die großen, z. T. gelapptkernigen Zellen, die allerdings meist auch nur unregelmäßig eingestreut waren und selten in einer kleinen Gruppe beisammenstanden, sowie die recht scharfe rundliche Abgrenzung des Knötchens, mit der Tendenz zentraler Abteilung, hoben diese Gebilde doch über ganz gewöhnliche entzündliche 
Veränderungen hinaus und verliehen ihnen schon etwas von granulomartigem Charakter. Etwas Typisches für Läsionen durch einen bekannten Erreger zeigten sie allerdings nicht. Die großen Zellen erinnerten z. T. an Epitheloide, besonders in den kleineren Formen. Zum Teil hatten sie, mit ihren öfters leicht lappigen Kernen eine gewisse Ähnlichkeit mit den Sternbergschen Zellen bei Lymphogranulomatose, irgendeinen bestimmteren Schluß ließ ihr Verhalten aber nicht zu.

Da auch klinisch jede bakteriologische Nachforschung negativ ausgefallen war, eröffneten sich uns vorerst auch von dieser Seite keine weiteren Anhaltspunkte und wir mußten die Diagnose offenlassen.

Während der folgenden Tage veränderte sich das Exanthem kaum. Es verschwanden einzelne Efflorescenzen, andere schossen dafür auf, der Charakter bleibt jedoch derselbe.

Die Frau starb unter septischen Erscheinungen und kam im hiesigen pathologischen Institut zur Sektion.

Dem Protokoll (Nr. 52/1918), für dessen Überlassung ich Herrn Professor Hedinger bestens danke, entnehme ich folgende Angaben:

Ziemlich großer Körper, von mittlerem Ernährungszustand. Livores hellrot. An der Streckseite der oberen Extremitäten kleine, braun verfärbte, leicht papulöse Efflorescenzen, ebenso an den Unterschenkeln, besonders rechts; über dem Gesäß mehr graurote kleinste Papeln. Am oberen Teil der Sternocleidomastoideusgegend kleine, derbe, unscharf begrenzte Tumoren zu fühlen. Zwerchfell beiderseits 4. Rippe. Unterer Leberrand in der Mittellinie $12 \mathrm{~cm}$ unter Ende Corp. sterni, in der r. Mam.-Linie $7 \mathrm{~cm}$ unter dem Rippenbogen. Milz ragt überall über den Rippenbogen links um ca. $1-6 \mathrm{~cm}$ hervor. Därme von mittlerer Füllung. Serosa glatt und glänzend. Appendix kurz, ganz in Adhäsionen eingebettet. In der Umgebung der Appendix, namentlich am Coecum hinten unten, multiple, teils in Gruppen gestellte miliare, weiße verkäste Knötchen. Lungen wenig retrahiert und kollabiert. Linke Lunge mit einzelnen leichten Adhäsionen und Verklebungen. Rechte Lunge frei. In den Pleurahöhlen je $50 \mathrm{ccm}$ blutige, trübe Flüssigkeit. Im Mediast. antic. wenig Fettgewebe und einzelne kleine, graurote Lymphdrüsen. Herz klein, schlaff, leicht dilatiert. Halsorgane: Zunge mit dickem, grauem Belag, Balgdrüsen der Zungenbasis, Tonsill. palat. nicht vergrößert. Am weichen Gaumen, an der Uvula, im Pharynx, bes. in den Sinus piriformes, auf der Epiglottis und im ganzen Larynx findet sich eine hochgradige Veränderung. Man sieht neben multiplen kleinen seichten scharfrandigen, vielfach zusammenfließenden, selten größeren, bis $1 \mathrm{~cm}$ messenden Geschwürchen, große Partien, besonders im Larynx eigentümlich weiß verfärbt, teils diffus, teils mehr herdweise, leicht erhaben. Die grauweißen Massen lassen sich leicht abstreifen; danach erscheint ein dunkelgraurotes Gewebe, in dem hier und da andeutungsweise feinste Knötchen von grauer Farbe liegen. Oesophagusschleimhaut blutreich. Schilddrüse, im linken Unterhorn ein $2 \mathrm{~cm}$ messender Kolloidknoten. Obere Cervicaldrüsen, besonders die in der Gegend des oberen Teils des Sternocleidomastoideus, auf beiden Seiten in eine derbe, $8: 4: 5 \mathrm{~cm}$ messende Geschwulst umgewandelt. Auf Schnitt sieht man in großer Zahl 2-5-20-30 mm messende, scharf begrenzte, gelbweiße, verkäste, derbe Herde, die durch graurotes derbes Gewebe mehr oder weniger scharf voneinander getrennt werden. Beim Ausschneiden des Sternocleidomastoideus findet man in dieser Höhe auch in ihm 
2-4 mm messende weiße, stark trübe Herde. Untere Cervicaldrüsen leicht vergrößert, ziemlich anthrakotisch, z. T. ebenfalls ausgedehnt verkäst, z. T. mit einzelnen miliaren trüben Knötchen. Linke Lunge voluminös, Luftgehalt überall gleichmäßig herabgesetzt. Pleura mit diinnem Fibrinbelag, subpleurale, besonders im vorderen Lappen multiple, z. T. in kleine Gruppen gestellte, miliare, verkäste Tuberkelknötchen. Auf Schnitt Unterlappen dunkelgraurot, von multiplen kleinsten 2--3 mm messenden grauweißen, gekömten, brïchigen lobulären Hepatisationen durchsetzt. Abstreifen läßt sich reichlich blutige, wenig schaumige, etwas trübe Flüssigkeit. Im Oberlappen läßt sich blutige, schaumige, in dünnen Schichten klare Flüssigkeit abstreifen. Gewebe nach dem Abstreifen glatt und glänzend, kompressibel, nur in den unteren hinteren Partien dieselben Herde wie im Unterlappen. Rechte Lunge ziemlich voluminös. Luftgehalt überall herabgesetzt, Pleura mit dünnem Fibrinbelag. Im Unterlappen läßt sich auf Schnitt abstreifen reichlich biutige, leicht triibe Flüssigkeit, Gewebe nach dem Abstreifen dunkelgraurot, durchsetzt von multiplen, etwas stark prominenten, kleinsten, graugelben lobulären Hepatisationen und vereinzelten, diffus verstreuten miliaren weißen Tuberkeln. Mittellappen: Abstreifen läßt sich schaumige, klare Flüssigkeit. Gewebe nach Abstreifen graurot, glatt und glänzend kompressibel. Im Gewebe ganz vereinzelt miliare weiße Tuberkel. Im Oberlappen hinten läßt sich reichlich blutige, schaumige, leicht trübe Flüssigkeit abstreifen. Gewebe nach dem Abstreifen graurot, durchsetzt von kleinsten multiplen $2--3 \mathrm{~mm}$ messenden, fast käsig aussehenden gelbweißen gekörnten Hepatisationen. Sonst Gewebe hellgraurot, glatt und glänzend, völlig kompressibel. Bronchialdrüsen mittelgroß, anthrakotisch. Milz $22: 12: 5 \mathrm{~cm}$. Kapsel etwas verdickt, Pulpa dunkelbraunrot, etwas vorquellend, Follikel klein, Trabekel deutlich. In der Pulpa zerstreut in mittlerer Zahl, z. T. in Gruppen gestellt, teils miliare, teils bis zu $2 \mathrm{~mm}$ messende weiße, trübe, derbe Knötchen. Nebennieren von mittlerer Größe in der linken ein 16: 10:10 mim messende grauroter, zentral gelegener Knoten; Rinde sonst fettarm, Mark wenig ausgesprochen. Nieren: mittlere Fettkapsel, fibröse Kapsel zart. Niere groß, sonst makroskopisch o. B. Leber groß, Oberfläche glatt: Serosa zart. Subserös springen ziemlich reichlich intensiv weiße, meist trübe, derbe Knötchen vor. Auf Schnitt acinöse Zeichnungen deutlich, grob, Zentra meist insulär, Peripherie etwas trübe. Vielfach auch im Zentrum ein kleinstpunktförmiger trüber Herd, Glissonsche Scheiden nicht verbreitert: Im Gewebe zerstreut ziemlich reichlich z. T. mehr gelbliche, meist aber weiße, miliare, selten 2-3 mm messende, derbe, etwas über die Schnittfläche prominierende Herdchen. Gallenblase o. B. Magen, Darm o. B. Auf der Serosa parietalis besonders über dem Zwerchfell und im Douglas und über dem Uterus reichlich miliare, vielfach in Gruppen gestellte, verkäste Knötchen. Retroperitoneale, mesenteriale, inguinale und axillare Lymphdrüsen klein, graurot. Wirbelkörper und Femur mit hellgraurotem Mark. Schädel o. B.

Diagnose: Chronische myeloide Leukämie, Dermatitis leucaemica, Tuberkulose der Cervicaldrüsen, chronische Tuberkulose der Pleura und des Peritoneums, Miliartuberkulose von Lungen, Milz und Leber, lobuläre Pneumonie aller Lappen, Pleuritis fibrinosa, Dilatatio cordis.

Hatten wir schon auf Grund des klinischen und histologischen Aussehens der Efflorescenzen uns mit der Annahme einer rein toxischen Ursache nicht recht zufrieden geben können, so erhob sich auf Grund des Sektionsbefundes sogleich die Frage, ob das Exanthem nun nicht als eine Teilerscheinung der allgemeinen Miliartuberkulose aufzufassen 
sei, da ja auch bei dieser mehrfach schon Exantheme beschrieben worden sind.

Bei Erwachsenen sind sie allerdings recht selten beobachtet worden und die mitgeteilten Fälle zeigen mit den unsrigen keine Ähnlichkeit. Es sind die von Goldscheider, Hedinger und Mibelli.

Goldscheider fand cutan und subcutan gelegene, mäßig prominierende, bläulichrote, schmerzhafte, verschwindende und neuentstehende Knoten, kurzdauernde Erytheme und einmal nur 48 Stunden bestehende flohstichähnliche Stippehen.

Bei Hedinger bestanden größere, cutan-subcutane, blaurote Infiltrationen mit zentraler Öffnung.

Mibelli sah auf dem Haarboden größere und kleinere Ulcera, nachdem anamnestisch auch am Körper furunkelähnliche Knoten aufgetreten und wieder verschwunden waren.

Andere Fälle die etwa als Miliartuberkulose angeführt werden, wie die von Nägeli, Vörner, Nobl, Bosselini, möchte ich hier nicht mit einbeziehen, da bei ihnen wohl eine Entstehung der Efflorescenzen auf dem Blutweg angegeben wird, eine allgemeine Miliartuberkulose aber nicht nachgewiesen wurde.

Etwas mehr Berührungspunkte im klinischen Aussehen unseres Falles ergeben sich dagegen mit den bei Kindern beschriebenen Miliartuberkulosen. Wenn auch bei ihnen, so wie sie z. B. von Jadassohn zusammengestellt sind, die Efflorescenzen z. T. noch recht verschieden aussehen, so finden sich doch einige Male kleine Papeln erwähnt. Z. B. bei Heller feinste rote Punkte und Knötchen, einzelne mit zentralem blassem Fleck, bei Leichtenstern mohnkorn- bis höchstens hanfkorngroße, lebhaft rote, derbe, kegelförmig zugespitzte Papeln, die sich auf vollständig normaler Haut mit scharfem Kontur steil erhoben, und bei Rensburg derbe, mohn- bis hanfkorngroße, rosa-blaßrote Knötchen.

Auch das von Leiner und spieler ziemlich genau umschriebene Bild der akuten hämorrhagischen Miliartuberkulose im Kindesalter hat gewisse ähnliche Züge, wenn sie es wie folgt beschreiben: „Das disseminiert an Stamm und Extremitäten, evtl. auch im Gesicht auftretende Exanthem hat im allgemeinen purpuraähnlichen Charakter. Die einzelnen Efflorescenzen sind durchsehnittlich stecknadelkopfgroß, kaum über das Niveau prominierend, lividrot bis rotbraun gefärbt, auf Fingerdruck nicht vollständig abblassend, zentral teils nur einen gelben Farbenton, teils eine kleine Delle, teils Krüstchen oder Schuppen zeigend. Sie sind ziemlich dicht gestellt, stellenweise zu kleinen Plaques konfluiert."

Die hellroten bis bräunlichroten, scharf umschriebenen Papeln, oft mit zentralem weißlichgelbem Fleck oder kleiner Hämorrhagie sind also mehrfach angegeben, auch ihre Gruppierung ist von Leiner und 
Spieler beobachtet worden. Von letzteren wird dazu besonders der hämorrhagische Charakter des Exanthems betont. Dieser trat auch bei uns in Erscheinung z. T. als kleine Blutungen im Zentrum der Papeln, vielmehr aber noch durch das gleichzeitige Vorhandensein zahlreicher Purpurafleckchen besonders am Stamm. Es wäre hier noch zu erwähnen, daß auch Purpura allein schon mehrfach ätiologisch mit Tuberkulose in Zusammenhang gebracht worden ist. Zuletzt hat Zieler die diesbezüglichen Angaben zusammengestellt. Für unser Thema sind unter diesen einige Fälle von Purpura des Unterschenkels bei Miliartuberkulose wichtig, die er selbst beobachtet hat, und ein Fall von Kluger, wo eine fulminante Purpura bei Miliartuberkulose auftrat.

Gewisse Ähnlichkeiten ließen sich also beim Vergleich unseres Exanthems mit manchen der bei akuter Miliartuberkulose der Kinder beobachteten Efflorescenzen schon feststellen. Die Histologie ließ uns dagegen in dieser Hinsicht völlig im Stich. Ebensowenig, wie wir in den intra vitam excidierten Stückchen irgendwelchen spezifischen tuberkuloiden Bau gefunden hatten, konnten wir in weiteren, der Leiche entnommenen, größeren Hautpartien Stellen finden, die einen solchen aufwiesen. In diesem Material traten sogar die Knötchen kaum mehr hervor; vorwiegend sah man nur die perivaseulären, und die etwas flächenhafter ausgeprägten, zellreicheren Infiltrate an den Papillarschlingen. Die großen Zellen der Knötchen hätte man vielleicht schon als etwas vergrößerte epitheloide Zellen ansehen können. Sie standen sogar hier und da in einer Gruppe von dreien oder fünfen locker beisammen. Um die ganzen Papeln aber im Sinne tuberkulöser Veränderungen deuten zu können, dazu waren sie doch nicht ausgeprägt genug, und viel zu unregelmäßig zerstreut.

An sich würde zwar ein unspezifisches Aussehen schließlich noch nicht viel zu sagen haben. Bei Miliartuberkulose sind ja auch ganz uncharakteristische Entzündungsherde und Nekrosen beschrieben worden (Leichtenstern, Rendsburg, Leiner und spieler). Dann aber waren stets massenhaft Tuberkelbacillen im Schnitt nachweisbar, während es uns, bei Durchmusterung von zahlreichen Schnitten, nicht gelang, auch nur einen solchen in einer Efflorescenz anzutreffen.

Wir müssen bier aber nochmals hervorheben, da $B$ unser histologisches Bild schon im ganzen uns doch nicht nur einem einfachen akut entzündlichen Prozeß zu entsprechen schien, und daß dann namentlich in den Efflorescenzen, die uns gerade für eine bakterielle Ursache fraglich waren, eine Struktur bestand, die nur als ein Ansatz zu einer Granulombildung angesprochen werden konnte. Für den Zusammenhang mit der Miliartuberkulose könnte dies doch nur im Sinne einer Abwehrreaktion des Körpers gedeutet werden. Der Organismus hätte somit den Tuberkelbacillen noch Widerstand geleistet. Deren Féhlen wäre 
also erklärlich. Dafür erhebt sich dann die Frage, warum es nicht zur Ausbildung deutlich ausgeprägter, tuberkuloider Strukturen gekommen ist, die sonst immer vorhanden sind, wo Tuberkelbacillen durch Antikörperwirkung zugrunde gehen. Die Antwort hierauf dürfte vielleicht darin zu finden sein, daß die Reaktionsweise des Körpers durch eine schon längere Zeit vorher bestehende Leukämie modifiziert worden sein kann.

Daß nicht nur die Haut, sondern auch der übrige Körper nicht wie ein nur von Miliartuberkulose befallener Organismus reagierte, dafür sprechen gleichfalls die histologischen Befunde an den inneren Organen.

Infolge freundlicher Úberlassung von Material dureh die pathologische Anstalt konnten wir von den verkästen Halsdrüsenpaketen und von der Milz Präparate selbst anfertigen. Bei Färbung mit Hämalaun-Eosin zeigen sich die Schnitte als vorwiegend aus großen nekrotischen, reichlich Kerntrümmer enthaltenden Herden bestehend, zwischen denen noch Reste der Grundsubstanz liegen, Fett und Bindegewebe mit Gefäßen und Nerven. Drüsensubstanz war in unseren Schnitten nicht mehr sichtbar. An sehr vielen Stellen grenzt die Nekrose unmittelbar an normales, unverändertes Bindegewebe. An anderen Stellen ist das benachbarte Gewebe mehr oder wenig ödematös gelockert und von einem verschiedenen dichten Zellinfiltrat durchsetzt, das sich hier und da als richtiger Saum an die Nekrose anlegt. Es besteht vorwiegend aus Rundzellen, z. T. Lymphocyten, z. T. Plasmazellen, z. T. Zellen mit gut gefärbtem Kern und deutlichem Protoplasmasaum, die als Myelocyten aufzufassen sind. Daneben finden sich Fibroblasten, sehr wenig zahlreiche Epitheloide, hier und da in einer Gruppe etwas gehäuft, und größere protoplasmareiche Zellen, oval, mit kleinem kompaktem Kern, die am ehesten wie sehr vergrößerte Plasmazellen aussehen, z. T. mit den großen Zellen in der Haut gewisse Ähnlichkeit haben. A usgeprägte Tuberkel finden sich nirgends. In den Nekrosen sind wenige Häufchen ziehlfester Stäbchen nachzuweisen.

Die Milz zeigt ein ganz verändertes Bild. Follikel finden sich nur ganz wenige, sehr kleine. Die Trabekel sind etwas verdickt. Die Pulpa zeigt die gewöhnliche Grundsubstanz, mit vermehrtem Blutgehalt und dicht erfüllt von Lymphocyten, Plasmazellen und Myelocyten. Tuberkel finden sich nicht, sondern nur einzelne kleine in die Pulpa eingestreute, scharf umsehriebene Nekroseherde, in denen nach Ziehl ebenfalls Tuberkelbacillen im Schnitt nachweisbar sind.

Nach dem Berichte der pathologischen Anstalt finden sich ferner in der Leber reichlich vielfach konfluierende, miliare Tuberkel, fast ausschließlich aus nekrotischem Gewebe bestehend, und nur selten an der Peripherie etwas verfettete Epitheloide, Langhanssche Riesenzellen und Lymphocyten aufweisend. 
Die größeren und die miliaren tuberkulösen Herde der inneren Organe bieten somit einen ebenfalls recht ungewöhnlichen Bau. Die in den Nekrosen gefundenen Tuberkelbacillen sind nicht derart zahlreich, als daß auf eine völlige Erschöpfung der Abwehrkräfte des Körpers geschlossen werden könnte, vielmehr dürfte auch in diesen Veränderungen. der Ausdruck der gleichen Modifikation der Reaktionsweise des Körpers durch die Leukämie gesehen werden, wie wir sie bereits zur Erklärung der Struktur der Hautefflorescenzen herangezogen haben.

Für letztere dürfte in bezug auf das Fehlen nachweisbarer Tuberkelbacillen in diesem Zusammenhang noch darauf hingewiesen werden, daß die Tuberkelbacillen in der Haut nach allgemeiner Annahme einen schlechteren Nährboden finden als in den inneren Organen, so daß sie sich nicht derart vermehren, um leicht nachgewiesen werden zu können. Um ihre Anwesenheit trotzdem noch beweisen zu können, dafür ist dann der Tierversuch von großem Wert. Dessen Ausfall bewies auch in unserem Fall, daß Tuberkelbacillen in der Haut vorhanden waren. Am 26. I. wurden einem Meerschweinchen mehrere kleine Papeln subcutan implantiert, einem zweiten wurde ein durch Zerreiben von solchen gewonnener Brei intraperitoneal injiziert. Das zweite Tier starb im August interkurrent, ohne Zeichen von Tuberkulose, das erstere dagegen verendete am 24. April an ausgesprochener Tuberkulose. Ein gleichzeitig mit Drüsenstückchen subcutan implantiertes Tier starb am 13. V., ein mit Milzsaft intraperitoneal gespritztes am 7. III., beide an Tuberkulose.

Natürlich ist mit diesem positiven Ausfall nicht bewiesen, daß Tuberkelbacillen gerade in einem der Knötchen gesessen haben. Die Möglichkeit, daß bei Miliartuberkulose zu jeder Zeit und an jeder Stelle der Haut ein Tuberkelbacillus liegen kann, ist nicht zu bestreiten. Wenn aber in der Haut entzündliche Veränderungen z. T. von annähernd granulomartigem Charakter bestehen, so ist es doch recht gezwungen, anzunehmen, sie seien nicht durch die Tuberkulosebacillen verursacht, sondern letztere hätten entweder zu Lebzeiten realktionslos in der Haut gelegen oder seien erst kurz vor dem Tode in sie gelangt. Immerhin geben wir zu, daß anch aus dem Tierexperiment ein eindeutiger, absolut beweisender Schluß ebensowenig wie aus unseren vorhergehenden Befunden gezogen werden kann.

Wir glauben daher das Resultat unserer Untersuchung nur dahin zusammenfassen zu können, daß das bei einer mit Miliartuberkulose komplizierten myeloiden Leukämie aufgetretene Exanthem in verschiedenen Beziehungen Ähnlichkeit mit Exanthemen zeigt, wie sie sowohl bei myeloider Leukämie, als auch bei Miliartuberkulose allein auftreten können. Die zahlreichen. Petechien, der doch mehrere Monate đauernde Verlauf mit anfänglich zeitweise starkem Jucken, sowie die 
histologisch einfach entzündlichen Erscheinungen würden eher zu ersterer passen, die papulösen Efflorescenzen dagegen mit ihrer eigenartigen Histologie dürften viel eher mit der Tuberkulose in Verbindung zu bringen sein, besonders wenn man eine, durch die Leukämie modifizierte Reaktionsfähigkeit des Körpers annimmt. Nicht unmöglich ist es, daß die ursprünglichen Efflorescenzen rein leukämische Petechien waren, wie ja noch viele sich fanden, daß aber dann in manchen von diesen Tuberkelbacillen bei ihrem Kreisen durch die Blutbahn sich festgesetzt und die weiteren Veränderungen erzeugt haben.

\section{Iiteratur.}

Arndt, Zur Kenntnis der leukämischen und aleukämischen Lymphadenose der Haut usw. Dermatol. Zeitschr. 18. 1911. - Bosellini, Tuberculosis cutanea pustulosa follicularis raf. Dieses Archiv 105, 180. 1910. - Bru usgaard, Über Hauteruptionen bei der myeloiden Leukämie und der malignen Granulomatose. Dieses Archiv 106. 1911. - Burckhardt, Zur Frage der akuten myeloiden Leukämie. Frankf. Zeitschr. f. Pathol. 6. 1911. - Goldscheider, Hautaffektionen bei der akuten Miliartuberkulose Monatshefte 1, 1882. Hedinger, Miliartuberkulose der Haut bei Tuberkulose der Aorta. Frankf. Zeitschr. f. Pathol. 2. 1909. - Hindenburg, Dtsch. Arch. f. klin. Med. 54. 1895. - Hirschfeld und Tobias, Zur Kenntnis der myelogenen Leukämie. Dtsch. med. Wochenschr. 1902, Nr. 6. - Jadasso hn, Die Tuberkulose der Haut. Mraceks Handb. d. Hautkrankh. 4, I. 1907. - Leiner und S pieler, Über disseminierte Hauttuberkulosen im Kindesalter, Ergebnisse der inneren Med. u. Kinderheilk. \%. 1911. - Lewa ndowsky, Die Tuberkulose der Haut. Springer. Berlin. 1916. - Mibelli, Disseminierte Miliartuberkulose des Haarbodens. Monatshefte 44. 1907. - Naegeli, Blutkrankheiten und Blutdiagnostik. 1912. - Naegeli, Über hämatogene Hauttuberkulose. Münch. med. Wochenschr. 1898. - Nobl, Zur Klinik and Histologie seltener Formen von Hauttuberkulose. Dieses Archiv 1900. Festschrift f. Kaposi, S. 849. - Paltauf, Die lymphat. Erkrankungen und Neubildungen der Haut. Mraceks Handb. d. Hautkrankh. 4, II. 1909. - Schlei p und Hildebrand, Münch. med. Wochenschr. 1905, Nr. 9. - Schultze, Ein Beitrag zur Kenntnis der akuten Leukämie. Zieglers Beiträge z. allg. Path. u. pathol. Anat. 39. 1906. - Vörner, Ein be merkenswerter Fall von tuberkulösem Hautexanthem. Münch. med. Wochenschr. 1906, Nr. 39. - Zieler, Hauttuberkulose und Tuberkulide. Jesionek, Ergebnisse. 1914, Bd. 3. 